\title{
MYCOCINOGENIC YEASTS ISOLATED FROM AMAZON SOILS OF THE MARACÁ ECOLOGICAL STATION, RORAIMA-BRAZIL
}

\author{
Marcos José Salgado Vital ${ }^{1,2}$; Jacqueline Abranches ${ }^{2}$; Allen Norton Hagler ${ }^{2}$; Lêda Cristina Mendonça-Hagler ${ }^{2 *}$ \\ ${ }^{1}$ Departamento de Biologia, Universidade Federal de Roraima, Boa Vista, RR, Brasil. ${ }^{2}$ Instituto de Microbiologia Prof. Paulo de \\ Góes, Departamento de Microbiologia Geral, Centro de Ciências da Saúde, Universidade Federal do Rio Janeiro, \\ Rio de Janeiro, RJ, Brasil
}

Submitted: December 18, 2001; Returned to authors for corrections: April 08, 2002; Approved: August 30, 2002

\begin{abstract}
The 240 yeasts isolated from soils of the Maracá Ecological Station in the Brazilian Amazon were identified and screened for mycocin production. These strains included $82 \%$ of ascomycetous and $18 \%$ basidiomicetous affinities and the prevalent species were Candida etchellsii, Candida famata, Candida robusta, Candida rugosa, Candida valida, Debaryomyces hansenii, Cryptococcus albidus, Cryptococcus laurentii, Rhodotorula glutinis, Rhodotorula minuta and Rhodotorula mucilaginosa. Mycocins able to kill some yeasts were produced by 6 strains identified as Issatchenkia sp., Saccharomyces exiguus?, Williopsis saturnus, var. subsufficiens, and 3 W. saturnus according to 26S rDNA D1/D2 region sequence and phenotypic data.
\end{abstract}

Key words: Mycocins, killer yeasts, tropical soil, Issatchenkia, Saccharomyces, Williopsis.

\section{INTRODUCTION}

Some yeasts strains produce and excrete extracellular toxins, mycocins, that are lethal to other sensitive yeast strains. Production of proteins with specific toxicity to related organisms, associated to specific immunity, is known to occur in the Ustilaginales, myxomycetes, paramecia and bacteria. The bacterial proteins with such capacity are denominated bacteriocins and similar compounds produced by yeasts have been called "killer" toxins but now are called mycocins and the strains that produce them as mycocinogenic (9). The $S$. cerevisiae mycocin system was first discovered in 1963 and has been the most studied. It is coded by a double stranded RNA gene that has been found only in Saccharomyces and the related genus Zygosaccharomyces (9,32). In other genera studied, the mycocins are coded in plasmidial DNA or chromosomal DNA, being activated at low $\mathrm{pH}$ and characterized as proteins or glicoproteins, with two or three subunits $(9,24)$. This natural phenomenon is a potential mechanism of competition by interference or amensalism, with the production of toxic compounds preventing access of sensitive yeasts to the resources and resulting in decreased population size of the less competitive species. Competition by interference generally occurs to obtain more resources by exclusion of other individuals or populations $(1,8,23)$. Mycocinogenic activity has been detected in 80 species distributed in 20 ascomycetous and basidiomycetous yeast genera. Most of the mycocinogenic yeasts are in the genera Candida, Cystofilobasidium, Cryptococcus, Debaryomyces, Filobasidium, Hansenula, Hanseniaspora, Kloeckera, Kluyveromyces, Metschnikowia, Pichia, Rhodotorula, Saccharomyces, Sporodiobolus, Williopsis and Zygosacharomyces (9). Mycocinogenic activity has been detected mainly in yeasts isolated from fruits, plant exudates, and plant structures, but rarely from soils $(3,23,25)$.

One of the most important conditions for detection of the mycocinogenic activity is the $\mathrm{pH}$ of the culture medium used for screening tests. This activity is generally expressed in acidic conditions between $\mathrm{pH} 3$ to 6 , with optimums ranging from 4 to 5 (33). Exceptions can be observed in mycocins that are inactivated by high temperature and tested at lower temperature

\footnotetext{
* Corresponding author. Mailing address: Instituto de Microbiologia Prof. Paulo de Goés, Departamento de Microbiologia Geral, CCS, UFRJ, 21944970, Rio de Janeiro, RJ, Brasil. Fax: (+5521) 2560-8028. E-mail: leda@ibpinet.com.br
} 
(13). Mycocins are more stable in solid media, because agitation can cause their inactivation (30). Glucose-peptone-yeast extractagar medium (GYP) or malt agar with phosphate-sodium citrate buffer is most often used for these tests. The relation between the inoculum sizes of the sensitive and mycocinogenic strains influences the test sensitivity. In some cases the addition of 10$15 \%$ glycerol increases the size of the inhibition zone, and facilitates interpretation $(9,13,17)$.

Mycocins are of industrial importance for microbiological control of fermentation processes, and as a model for the study of secretion mechanisms involving extracellular proteins and glicoproteins (17). The application of mycocinogenic yeasts in the fermentation and beverage industry has grown with the objective of eliminating wild strains responsible for product deterioration and production of undesirable compounds $(18,32)$. The mycocins have only antifungal activity and are inactive against bacteria and protozoans (9). Some mycogenic strains act against pathogenic yeasts, such as Candida albicans and Candida glabrata, and filamentous fungi, suggesting potential application against fungal infections $(14,20,28)$. Our objective was to detect 'in vitro' mycocin producing yeasts from Amazon soils, and identify these strains by conventional and molecular methods.

\section{MATERIALS AND METHODS}

Yeasts were isolated from soil samples collected from the Maracá Ecological Station, located on Maracá Island $130 \mathrm{~km}$ from Boa Vista, in the Amazon state of Roraima, Brazil. The Ultisoils are the predominant soils on the Island, especially oxissoil, with the occurrence of red-yellow ultisoils, in euthrophic and distrophic forms. Yeast isolations were done by the spread plate method on acidified Y-M Agar medium $(0.3 \%$ yeast extract, $0.3 \%$ malt extract, $0.5 \%$ peptone, $1 \%$ glucose and $2 \%$ agar) with $200 \mathrm{mg} / \mathrm{L}$ of chloramphenicol, $0.15 \%$ sodium propionate, and $\mathrm{pH}$ adjusted to 4.5 with $\mathrm{HCl}$ and incubated 3 to 5 days at $25 \pm 3^{\circ} \mathrm{C}$ $(2,22)$. Selected colonies were streaked on $\mathrm{Y}-\mathrm{M}$ agar to obtain pure cultures and maintained at $8 \pm 4^{\circ} \mathrm{C}$ on $2 \%$ glucose- $0.5 \%$ yeast extract- $2 \%$ malt extract- $0.1 \%$ monosodium phosphate $2 \%$ agar slants covered with sterile mineral oil.

Mycocinogenic activity was tested in triplicate, on YM agar medium supplemented with $0.003 \%$ of methylene blue and $15.0 \%$ of glycerol and buffered with $0.01 \mathrm{M}$ citrate buffer to $\mathrm{pH} 4.2$. The strains $C$. glabrata Y-55 (NCYC 388, IMUFRJ 50.083) and Pichia heedii 83.504-2, sensitive to the majority of the known mycocins, were grown for $24 \mathrm{~h}$ at $21^{\circ} \mathrm{C}$ on $\mathrm{YM}$ agar, then were suspended in distilled water at $4 \times 10^{5} \mathrm{cells} / \mathrm{ml}$ and spread on the medium surface with sterile swabs. The yeasts that were tested for mycocin production were grown in the same way and inoculated in streaks on medium after inoculation with the sensitive strain. The plates were incubated at $23^{\circ} \mathrm{C}$ and were observed daily for 3 days. Isolates were considered as mycocinogenic if they produced an inhibition zone with no growth and adjacent blue zone indicating cellular death of the sensitive strain. Strains of Pichia kluyveri (IMUFRJ 51.498) and $P$. ohmeri-like (IMUFRJ 51.535) were employed as positive controls with mycocinogenic activity. The confirmation of activity was obtained through the replica-plating technique in which the sensitive test strains were incorporated individually into the agar medium and 25 strains to be tested for mycocin production were then inoculated onto each plate. The incubation conditions, positive controls and evaluation were the same as noted above. To test the ability of mycocinogenic yeasts to eliminate yeasts from soil communities, 121 selected strains were used as lawns to determine the sensitivity to the 6 toxinproducing yeasts.

The phenotypic characterization and identification of yeast cultures was done according to Kurtzman and Fell (11) and Barnett et al. (5). Representative isolates of different species were characterized by the size of the ITS region and the isolates producing mycocins also by sequencing the D1/D2 region of the rDNA large subunit $(7,10,12,26,29)$. DNA was extracted from pure cultures and amplified by PCR using the universal primers ITS 1 and ITS 4 or NL-1 and NL-4. Extracted DNA was purified for sequencing using the Concert Rapid PCR purification System (Gibco BRL), sequencing done using the Big Dye Termination Cycle Sequencing kit (ABI PRISM) and GeneAmp 9700 program according to the manufacturer's recommendations. Electrophoresis of the sequence reaction mixtures was done with an automatic DNA sequencer (ABI PRISM model 310 Genetic Analyzer). The sequence was edited using the DNASIS for Windows (version 2.1) software and aligned by the ClustalW program. Yeasts were identified using BLAST analysis and the GeneBank database. The designation of species followed by the suffix "-like" indicates that the organism is similar to this species but sufficiently different to be a new species, whereas the suffix "?" indicates the yeast appears to be of the named species but the identification is uncertain.

\section{RESULTS}

The 240 yeast isolates were grouped into 16 genera and 66 species, with $82 \%$ of the strains having ascomycetous affinity (Table 1). Only 6 strains, all of ascomycetic affinity had micocinogenic activity with the same profiles against both the $C$. glabrata and $P$. heedii test strains. These included Issatchenkia sp. (probable teleomorph of Candida pseudolambica), Saccharomyces exiguus ?, W. saturnus var. subsufficiens, and 3 W. saturnus. The activity of the 6 mycocinogenic strains against yeasts from the soil community is presented in Table 1. Issatchenkia sp., and W. saturnus were isolated from samples collected during the dry season, whereas $S$. exiguus? and $W$. saturnus var. subsufficiens were collected during the rainy season. 
Table 1. Amazon forest soil yeasts and the number of them that were sensitive to mycocins produced by six of the isolates.

\begin{tabular}{|c|c|c|c|c|c|c|c|}
\hline Yeasts & $\begin{array}{c}\text { Number of } \\
\text { isolates }\end{array}$ & $\begin{array}{c}W . \text { saturnus } \\
\text { RR41 }\end{array}$ & $\begin{array}{c}\text { W. saturnus } \\
\text { RR14 }\end{array}$ & $\begin{array}{c}\text { W. saturnus } \\
\text { RR19 }\end{array}$ & $\begin{array}{c}\text { W. saturnus } \\
\text { RR177 }\end{array}$ & $\begin{array}{c}\text { Issatchenkia } \\
\text { sp. }\end{array}$ & S. exiguus? \\
\hline ASCOMYCETOUS & & $\mathrm{a}(\mathrm{b})$ & & & & & \\
\hline Candida spp. (9 species) & 10 & $0(8)$ & $0(8)$ & $0(8)$ & $0(8)$ & $0(8)$ & $0(8)$ \\
\hline Candida bombicola & 2 & $0(2)$ & $0(2)$ & $0(2)$ & $0(2)$ & $0(1)$ & $0(1)$ \\
\hline Candida colliculosa & 5 & $0(3)$ & $0(3)$ & $0(3)$ & $0(3)$ & $0(1)$ & $0(1)$ \\
\hline Candida edax? & 2 & $0(2)$ & $0(2)$ & $0(2)$ & $0(2)$ & $0(2)$ & $0(2)$ \\
\hline Candida famata-like & 2 & $0(2)$ & $0(2)$ & $0(2)$ & $0(2)$ & $0(2)$ & $0(2)$ \\
\hline Candida gropengiesseri? & 1 & $1(1)$ & $1(1)$ & $1(1)$ & $1(1)$ & $0(1)$ & $0(1)$ \\
\hline Candida lusitaniae & 1 & $1(1)$ & $1(1)$ & $1(1)$ & $1(1)$ & $1(1)$ & $1(1)$ \\
\hline Candida holmiii & 1 & $0(1)$ & $0(1)$ & $0(1)$ & $0(1)$ & $0(1)$ & $0(1)$ \\
\hline Candida holmiii-like & 1 & $1(1)$ & $1(1)$ & $1(1)$ & $1(1)$ & $0(1)$ & $0(1)$ \\
\hline Candida oleophila & 4 & $1(2)$ & $1(2)$ & $1(2)$ & $1(2)$ & $0(2)$ & $0(2)$ \\
\hline Candida schatavii? & 1 & $0(1)$ & $0(1)$ & $0(1)$ & $0(1)$ & $0(1)$ & $0(1)$ \\
\hline Candida sorbophila & 1 & $0(1)$ & $0(1)$ & $0(1)$ & $0(1)$ & $0(1)$ & $0(1)$ \\
\hline Candida tannotolerans? & 1 & $0(1)$ & $0(1)$ & $0(1)$ & $0(1)$ & $0(1)$ & $0(1)$ \\
\hline Candida valida & 7 & $1(3)$ & $1(3)$ & $1(3)$ & $1(3)$ & $1(3)$ & $1(3)$ \\
\hline Candida versatilis -like & 1 & $0(1)$ & $0(1)$ & $0(1)$ & $0(1)$ & $0(1)$ & $0(1)$ \\
\hline Debaryomyces hansenii & 15 & $4(4)$ & $4(4)$ & $4(4)$ & $4(4)$ & $0(4)$ & $0(4)$ \\
\hline Debaryomyces occidentalis & 4 & $1(2)$ & $1(2)$ & $1(2)$ & $1(2)$ & $1(2)$ & $1(2)$ \\
\hline Debaryomyces vanrijiae & 5 & $0(2)$ & $0(2)$ & $0(2)$ & $0(2)$ & $0(2)$ & $0(2)$ \\
\hline Geotrichum candidum & 2 & $1(1)$ & $1(1)$ & $1(1)$ & $1(1)$ & $0(1)$ & $0(1)$ \\
\hline Issatchenkia occidentalis & 3 & $0(2)$ & $0(2)$ & $0(2)$ & $0(2)$ & $0(2)$ & $0(2)$ \\
\hline Issatchenkia orientalis & 1 & $1(1)$ & $1(1)$ & $1(1)$ & $1(1)$ & $0(1)$ & $0(1)$ \\
\hline Issatchenkia sp. & 1 & $1(1)$ & $1(1)$ & $1(1)$ & $1(1)$ & $0(1)$ & $0(1)$ \\
\hline Lipomyces tetraporus & 3 & $0(1)$ & $0(1)$ & $0(1)$ & $0(1)$ & $0(2)$ & $0(2)$ \\
\hline Torulaspora delbruecki & 3 & $1(2)$ & $1(2)$ & $1(2)$ & $1(2)$ & $0(1)$ & $0(1)$ \\
\hline Williopsis saturnus RR41 & 1 & $0(1)$ & $0(1)$ & $0(1)$ & $0(1)$ & $0(1)$ & $0(1)$ \\
\hline Williopsis saturnus & 3 & $0(3)$ & $0(3)$ & $0(3)$ & $0(3)$ & $0(3)$ & $0(3)$ \\
\hline Zygosaccharomyces sp. & 2 & $0(2)$ & $0(2)$ & $0(2)$ & $0(2)$ & $0(2)$ & $0(2)$ \\
\hline \multicolumn{8}{|l|}{ BASIDIOMYCETOUS } \\
\hline Cryptococcus albidus & 8 & $1(4)$ & $1(4)$ & $1(4)$ & $1(4)$ & $0(4)$ & $1(4)$ \\
\hline Cryptococcus curvatus & 2 & $0(2)$ & $0(2)$ & $0(2)$ & $0(2)$ & $0(2)$ & $0(2)$ \\
\hline Cryptococcus hungaricus & 3 & $0(2)$ & $0(2)$ & $0(2)$ & $0(2)$ & $0(2)$ & $0(2)$ \\
\hline Cryptococcus laurentii & 11 & $0(4)$ & $0(4)$ & $0(4)$ & $0(4)$ & $0(4)$ & $0(4)$ \\
\hline Fellomyces fuzhouensis & 1 & $0(1)$ & $0(1)$ & $0(1)$ & $0(1)$ & $0(1)$ & $0(1)$ \\
\hline Rhodotorula aurantiaca & 1 & $0(1)$ & $0(1)$ & $0(1)$ & $0(1)$ & $0(1)$ & $0(1)$ \\
\hline Rhodotorula glutinis & 14 & $0(4)$ & $0(4)$ & $0(4)$ & $0(4)$ & $0(4)$ & $0(4)$ \\
\hline Rhodotorula minuta & 7 & $0(2)$ & $0(2)$ & $0(2)$ & $0(2)$ & $0(2)$ & $0(2)$ \\
\hline Rhodotorula mucilaginosa & 27 & $0(5)$ & $0(5)$ & $0(5)$ & $0(5)$ & $0(5)$ & $0(5)$ \\
\hline Sporobolomyces roseus & 2 & $0(2)$ & $0(2)$ & $0(2)$ & $0(2)$ & $0(2)$ & $0(2)$ \\
\hline Sporobolomyces shibatanus & 2 & $1(2)$ & $1(2)$ & $1(2)$ & $1(2)$ & $1(2)$ & $1(2)$ \\
\hline Trichosporon asteroides & 1 & $0(1)$ & $0(1)$ & $0(1)$ & $0(1)$ & $0(1)$ & $0(1)$ \\
\hline Trichosporon ovoides & 4 & $0(1)$ & $0(1)$ & $0(1)$ & $0(1)$ & $0(1)$ & $0(1)$ \\
\hline
\end{tabular}

$a=$ number of sensitive strains; $b=$ number of tested strains. 
The Williopsis strains showed mycocinogenic activity against many yeasts from their soil habitat ( $48 \%$ of all strains, $36 \%$ of ascomycetous species, $14.5 \%$ of basidiomycetous species) compared with S. exiguus ? (19\% of all strains, $16.4 \%$ of ascomycetous species and $15 \%$ of basidomycetous species) and Issatchenkia sp. (9.5\% of all strains, $14.5 \%$ of ascomycetous species and $8 \%$ of basidomycetous species). The 4 Williopsis strains presented equal activity profiles in relation to the species of sensitive strains, producing growth inhibition and cellular death zones of about $12 \mathrm{~mm}$ diameter. The activity of Issatchenkia sp. and S. exiguus?, produced inhibition and death in a 10-mm diameter zone. All of these mycocinogenic strains fermented glucose, 2 had low carbon source assimilation profiles with less than 9 positive results and 4 had intermediate assimilation profiles of 11 to 19 out of the 37 carbon sources tested. Strain RR115 failed to show the latent growth on glucosamine characteristic of $C$. pseudolambica, but otherwise was consistent with the description of this species and formed ascospores typical of the genus Issatchenkia. Strain RR169 fit the description of S. exiguus, the teleomorph of Candida holmii.

The ITS region length of Issatchenkia sp., of about 430 bp, and S. exiguus ?, $700 \mathrm{bp}$, were consistent with those of similar species, while $W$. saturnus and like strains, with fragments of $600 \mathrm{bp}$, were compatible with type strain (IMPPG 51.700). The D1/D2 region nucleotide sequence from Issatchenkia sp. was $99 \%$ similar (527/530bp) to that of C. pseudolambica (GenBank U71063), indicating that it should represent the teleomorph of this species. That of strain RR169 had 90\% similarity (253/279bp) with S. exiguus (GenBank U68553) meaning that it should be closely related. The pairing of D1/D2 region of 26 rDNA of W. saturnus var. subsufficiens RR41 with Gene Bank data for the type strain (U75960), resulted in 98.2\% (487/496bp) similarity; while the sequences of $W$. saturnus RR14 showed 85.4\% (287/336), W. saturnus RR19 showed 92.4\% (340/368bp), and W. saturnus RR177, presented $91.1 \%$ (224/246bp) similarity with the sequence of the type strain.

\section{DISCUSSION}

The frequency of mycocinogenic strains isolated from natural environments varies for different microhabitats but is greater than that found among isolates from culture collections $(23,24)$. Most of the reports of mycocinogenic yeasts in natural environments, including tropical ones, refer to ephemeral habitats like fruits with high concentrations of sugar, and associated insects (3). The percentage [and isolated strains] of mycocin producing yeast species in communities found in tropical fruits was $24 \%$ of the species [13\% of strains] for Amapa fruit, $27 \%$ of the species [ $10 \%$ of strains] and for guava from a forest area, and for Cactus stem necrosis $12 \%$ of the species [6.4\% of strains] $(1,3)$. In association with animals mycocinogenic yeasts from the marsupial Didelphis marsupialis fecal pellets
$6.9 \%$ of the species [10\% of strains], Drosophila $18 \%$ of the species [9\% of strains] and mangrove invertebrates $15 \%$ of the species [27\% of strains], but for yeasts from the marsupial Philander frenata and rodent fecal pellets mycocins were not detected $(1,2,4$, and unpublished data) in their yeast communities. Mycogenic yeasts in water from bromeliad tanks had $5 \%$ of the species [ $2 \%$ of strains] and from a swamp water $8 \%$ of the species [ $8 \%$ of strains] (unpublished data). In most of the habitats mycocinogenic yeasts were not among the prevalent species. However, in some cases such as those of $K$. aestuarii in mangrove invertebrates, $P$. kluyveri in cactus necrosis and guava fruits and W. saturnus in swamp and bromeliad waters they were among the prevalent yeasts. In Amazon soils we found the percentage of mycocinogenic species to be most similar to that found in bromeliad tank and swamp waters in previous studies using similar methodology of detection. Soils are generally more stable and lower nutrient level habitats than fruits, and should favor less fermentative and broader assimilation profile species. However, they are not at all homogenous in nature, but a mosaic of microhabitats some of which can be nutrient rich such as dead plant or animal matter. In the lower nutrient level habitats, fast growth and elimination of nutrient competitors may not be as much of an advantage in soils when there is not much to compete for and mycocinogenic species would use some energy making toxins. Other factors allowing persistence with lower levels of more diverse nutrient resources could have more importance in habitats like soil.

In the present work we used two phylogenetically distant strains, which were sensitive to the majority of the described toxins, and we found a similar diversity of mycocinogenic yeasts to that obtained in other studies using only 1 or 2 sensitive strains $(1,3,24)$. The utilization of more than one sensitive strain to detect mycocin production has been indicated in order to detect a greater number of mycocinogenic isolates $(9,24,32)$. The use of a larger number of more diverse test strains applied to isolates of more diverse origin should reveal more cultures with this activity. This was the case for a collection of isolates from diverse substrates of the Brazilian Atlantic Forest tested for mycocin production against a set of yeasts of industrial or medical importance (6). It is also probable that the basidiomycetous yeasts are more important in the tropical soil habitat than indicated by our data because the culture medium and incubation conditions used in this study were more favorable for the isolation of ascomycetous yeasts.

Sequence analysis of the D1/D2 region of the 26SrDNA gene with about 550 base pairs has been shown by Kurtzman and Robnett (12) to allow identification of most ascomycetous yeast species and their anamorphs. Strains that show more than $1 \%$ of nucleotides substitution in this region are probably different species, while strains with substitutions in up to 3 nucleotides (about $0.5 \%$ ) belong to a same species or a closely related one. Comparison of sequences of this region of type 
and authentic cultures deposited in the GenBank (12) allow identification in most cases of described yeasts to the species level and can show the closest species affinity of undescribed species. Spore production and the alignment of D1/D2 sequence from strain RR115 indicated it is the teleomorph of $C$. pseudolambica so it has been designated as Issatchenkia sp. and its phenotypic characteristics are in agreement with this identification (21). Our partial D1/D2 sequence data suggest that strain RR169 may be a new species similar to S. exiguus of the "sensu lato" group so it has been designated here as $S$. exiguus? although its phenotypic characteristics are typical of this species. A new species with high phenotypic similarity to existing Saccharomyces species has been isolated previously from Brazilian forest microhabitats (15). W. saturnus includes several varieties reflecting a well documented high level of variation within this genetically related group, but without delineation among them at the species level. Strain RR41 had a high sequence similarity, when compared with the type culture of W. saturnus var. subsufficiens and was also consistent in phenotypic characteristics with this organism. However strains RR14, RR19, and RR177 had an intermediate level of partial D1/ D2 sequence similarity but based on incomplete sequences of the region. They are closely related to W. saturnus and have a broad mycocin activity profile which is typical of this species, but do not fit any of the existing five varieties. All 4 of the Willopsis isolates were in the same clade, but 3 may be distinct from existing varieties of W. saturnus.

W. saturnus is known to have a relatively broad spectrum of mycocinogenic activity and is a cosmopolitan species, frequently isolated from soil and related habitats $(4,11,16,31)$. The presence in soil at relatively high frequency of isolation and mycoinogenic properties we found for this group are consistent with previous reports. Candida pseudolambica, the probable anamorph of Issatchenkia sp RR 115, has been found to occur in stools, forages and temperate climate soils and a similar yeast has been isolated from mangrove invertebrates (4), but has not been found previously in tropical soils. $S$. exiguus, and its anamorph $C$. holmii, has a record of occurrence in soils, fruits, juices of fruits and products of fermentation, preserved vegetables, in temperate environments $(5,11)$ and has been reported from a tropical mangrove (4) Brazilian Atlantic Forest marsupial fecal pellets (2) and polluted estuarine waters (22). There is no record in the literature of mycocinogenic activity for $S$. exiguus nor for this Issatchenkia sp, but the genus Issatchenkia is part of the P. membranifaciens clade, which includes many mycocin producers as does the Saccharomyces clade, so the presence of this characteristic could be expected in these species. The 4 Williopsis isolates had identical results of activity against other strains from the soil community but $S$. exiguus? and Issatchenkia sp. were different from these and from each other in this activity suggesting they may produce different toxins.
The presence of non-mycocynogenic strains of species reported as including producers such as $C$. famata, $R$. mucilaginosa, Cr. albidus and Cr. laurentii, demonstrates the variability of this characteristic. This could result from poor taxonomic delineation of these species in studies relying mostly on a few differences in biochemical tests for identification, or genetic variation among strains. For example, our $C$. famata isolates varied in sensitivity to mycocins. This species is poorly delineated by conventional taxonomic methods so much of the data attributed to this species may be for other misidentified or undescribed species that can fall within its broad phenotypic description. Mechanisms of control and expression of mycocin production for the majority of the known strains, and sensitivity and resistance relationships among strains needs further study (9). Different levels of sensitivity to mycocynogenic activity have been used as a factor for intraspecific distinction in $C$. albicans and S. cerevisae $(19,27)$.

Mycocinogenic yeasts have a broad geographic distribution in diverse microhabitats, although they generally make up a relatively small portion of their yeast communities. Our preliminary data indicate the yeast community in Amazon soils to be diverse. We have shown the presence of mycocinogenic yeasts in a limited collection of yeasts from soils in a small Amazon region. Further studies of yeasts in tropical soils should allow discovery of new species and novel mycocinogenic yeasts.

\section{ACKNOWEDGEMENTS}

We thank W. T. Starmer for sending his mycocin sensitive strain $P$. heedii 83504-2. Financial support was provided by PRONEX, CAPES, FAPERJ and CNPq. We are grateful to IBAMA for access to Maracá Island, and to Anderson Cabral and Olavo Jacinto do Nascimento for technical support.

\section{RESUMO}

\section{Leveduras micocinogênicas de solos da Estação Ecologica de Maracá, Roraima-Brasil}

Duzentos e quarenta linhagens de leveduras foram isoladas de amostras de solos da Estação Ecológica de Maracá, na Amazônia Brasileira, as quais representam $82 \%$ de leveduras de afinidade ascomicética e 18\% basidiomicética. As especies dominantes foram Candida etchellsii, Candida famata, Candida robusta, Candida rugosa, Candida valida, Debaryomyces hansenii, Cryptococcus albidus, Cryptococcus laurentii, Rhodotorula glutinis, Rhodotorula minuta and Rhodotorula mucilaginosa. A capacidade das leveduras produzirem e excretarem toxinas letais a cepas sensíveis de leveduras, atividade micocinogênica, foi investigada. Seis 
linhagens foram capazes de produzir micocinas: Issatchenkia sp., Saccharomyces exiguus?, Williopsis saturnus var. subsufficiens, e $3 \mathrm{~W}$. saturnus identificadas conforme os dados de taxonomia molecular baseados nas sequiências da região D1/D2 do 26S rDNA.

Palavras-chave: micocinas, leveduras "killer", solos tropicais, Issatchenkia, Saccharomyces, Williopsis.

\section{REFERENCES}

1. Abranches, J.; Morais, P.B.; Rosa, C.A.; Mendonça-Hagler, L.C.; Hagler, A.N. The incidence of killer activity and extracellular proteases in tropical yeast communities. Can. J. Microbiol., 43: 328-336, 1997.

2. Abranches, J.; Valente, P.; Nóbrega, H.N.; Fernandez, F.A.S.; MendonçaHagler, L.C.; Hagler, A.N. Yeast diversity and killer activity in fecal pellets from marsupials and rodents in a brazilian tropical habitat mosaic. FEMS Microbiol. Ecol., 26: 27-33.

3. Abranches, J.; Vital, M.J.S.; Starmer, W.T.; Mendonça-Hagler, L.C.M.; Hagler, A.N. The yeast community and mycocin producers of guava fruit in Rio de Janeiro, Brazil. Mycologia, 92:16-22, 2000.

4. Araujo, F.V.; Soares, C.A.G.; Hagler, A.N.; Mendonça-Hagler, L.C. Ascomycetous yeast communities of marine invertebrates in a Southeast Brazilian mangrove ecosystem. Antonie van Leeuwenhoek, 68: 91-99, 1995.

5. Barnett, J.A.; Payne, R.W.; Yarrow, D. Yeast: Characteristics and Identification. $2^{\text {nd }}$ ed. Cambridge University Press, Cambridge. 1024p., 1990.

6. Buzzini, P.; Martini, A. Biodiversity of killer activity isolated from a Brazilian rain forest. Can. J. Microbiol., 46: 607-611, 2000.

7. de Barros Lopes M.; Soden, A.; Martens, A.L.; Henschke, P.A.; Langridge, A. Differentiation and species identification of yeasts using PCR. Int. J. Syst. Bacteriol., 48: 279-286, 1998.

8. Ganter, P.F.; Starmer, W.T.; Killer factor as a mechanism of interference competition in yeasts associated with cacti. Ecology, 73: 54-67, 1992.

9. Golubev, W.I. Mycocins (Killer Toxins). In: Kurtzman, C.P.; Fell, J.W. (eds.) The Yeasts, A Taxonomic Study. $4^{\text {rd }}$ ed. Elsevier Science B.V., Amsterdan, 1998. 55-62.

10. Kurtzman, C.P. Prediction of biological relatedness among yeasts from comparisons of nuclear DNA complementarily. Studies in Mycology, 30: 458-456, 1987.

11. Kurtzman, C.P.; Fell, J.W. The Yeasts, A taxonomic Study. $4^{\text {rd }}$ ed. Elsevier Science B.V., Amsterdan, 1998.

12. Kurtzman, C.P.; Robnett, C.J. Identification and phylogeny of ascomycetous yeast from analysis of nuclear large subunit (26S) ribosomal DNA partial sequences. Antonie van Leeuwenhoek, 73: 331-371, 1998.

13. Lehmann, P.F.; Lemon, M.B.; Ferencak, W.J. Antifungal compounds ("killer factors") produced by Kluyveromyces species and their detection on an improved medium containing glycerol. Mycologia, 79: 790-794, 1987.

14. Middelbeek, E.J.; Hermans, J.H.; Stum, C.; Muytjens, H.L. High incidence of sensitivity to killer toxins among Candida and Torulopsis isolates of human origin. Antim. Agent Chem., 17: 350-354, 1980.

15. Naumov, G.I.; James, S.A.; Naumova, E.; Louis, E.J.; Roberts, I.N Three new species in the Saccharomyces senso stricto complex:
Saccharomyces cariocanus, Saccharomyces kudriavzevii and Saccharomyces mikatae. Int. J. Syst. Evol. Microbiol., 50: 19311934, 2000.

16. Ohta, Y.; Tsukada, Y.; Sugimori, T. Production, purification and characterization of HYI, an anti-yeast substance, produced by Hansenula saturnus. Agric. Biol Chem., 48: 903-908, 1984.

17. Panchal, C.J.; Meacher, C.; van Oostrom, J.; Stewart, G.G. Phenotypic expression of Kluyveromyces lactis killer toxin against Saccharomyces spp. Appl. Environ. Microbiol., 50: 257-260, 1985.

18. Pfeiffer, P.; Radler, F.; Caspritz, G.; Hanel, H. Effect of a killer toxin of yeast on eucaryotic systems. Appl. Environ. Microbiol., 54: 10681069, 1988.

19. Polonelli, L.; Archibusacci, C.; Sestito, C.; Morace, G. Killer system: A simple method for differentiating Candida albicans strains. $J$. Clin. Microbiol., 17: 774-780, 1983.

20. Polonelli, L.; Lorenzini, R.; De Bernardis, F.; Morace, G. Potential therapeutic effect of yeast killer toxin. Mycopathologia, 96: 103107, 1986.

21. Smith, M.T.; Poot, G.A.; Kull, T. Segregation of Candida pseudolambica sp. nov. from Candida lambica and closely related species by deoxyribonucleic acids relatedness. Studies in Mycology, 31: 171176,1989

22. Soares, C.A.G.; Araujo, F.V.; Hagler, N.A.; Mendonça-Hagler, L.C.; Hagler, A.N. Ascomycetos yeasts from tropical intertidal dark mud of Southeast Brazilian estuaries. J. Gen. Appl. Microbiol., 43: 265272, 1997

23. Starmer, W.T.; Ganter, P.F.; Aberdeen, V. Geographic distribution and genetics of killer phenotypes for the yeast Pichia kluyveri across the United States. Appl. Environ. Microbiol., 58: 990-997, 1992.

24. Starmer, W.T.; Ganter, P.F.; Aberdeen, V.; Lachance, M.-A.; Phaff, H.J. The ecological role of killer yeasts in natural communities of yeasts. Can. J. Microbiol., 33: 783-796, 1987.

25. Stumm, C.; Hermans, J.M.H.; Middelbeek, E.J.; Croes, A.F.; De Vries, G.J.M.L. Killer-sensitive relationships in yeasts from natural habitats. Antonie van Leeuwenhoek, 43: 125-128, 1977.

26. Valente, P.S.; Gouveia, F.C.; Lemos, G.A.; Pimentel, D.; van Elsas, J.; Mendonça-Hagler, L.C.; Hagler, A.N. PCR amplification of the rDNA Internal Transcribed Spacer region for differentiation of Saccharomyces cultures. FEMS Microbiol. Lett., 137: 253-256, 1996.

27. Vaughan-Martini, A.; Cardinali, G.; Martini, A. Differential killer sensitivity as tool for fingerprinting wine-yeast strain of Saccharomyces cerevisiae. J. Ind. Microbiol. Biotechnol., 17: 124-127, 1996.

28. Walker, G.M.; McLeod, A.H.; Hodgson, V.J. Interactions between killer yeasts and pathogenic fungi. FEMS Microbiol. Lett., 127: 213222, 1995

29. White, T.J.; Bruns, T.; Lee, S.; Taylor, J. Amplification and direct sequence of fungal ribosomal RNA genes for phylogenetics. In: PCR protocols: A Guide to Methods and Applications, 1990.

30. Wilson, C.; Whittaker, P.A. Factors affecting activity and stability of the Kluyveromyces lactis killer toxin. Appl. Environ. Microbiol., 55: 695-699, 1989

31. Yamamoto, T.; Hiratani, T.; Hirata, H.; Imai, M.; Yamaguchi, H. Killer toxin from Hansenula mrakii selectively inhibits cell wall synthesis in a sensitive yeast. FEBS Lett., 197: 50-54, 1986.

32. Young, T.W. Killer yeasts. In: Rose, A.H.; Harrisson, J.S. (eds.). The Yeasts. V. 2. Acad. Press, London, p.131-164, 1987.

33. Young, T.W.; Yagiu, M. A comparison of the killer character in different yeasts and its classification. Antonie van Leeuwenhoek, 44: 59-77, 1978. 